\section{Justice through the J88: The doctor's role in the criminal justice system}

To the Editor: South Africa has an unacceptably high rate of interpersonal violence. ${ }^{[1]}$ Recent well-publicised incidents have prompted South Africans to re-examine the massive problem of violence against women in the country. ${ }^{[2]}$ As healthcare practitioners, many South African doctors are regularly exposed to victims of violent crime and are responsible for their care. For those victims who wish to make a case, the $J 88$ form serves as a crucial piece of medical evidence. ${ }^{[3]}$ It is an official form issued by the Department of Justice which documents the medico-legal examination that the healthcare practitioner performs on a victim and highlights findings that are potentially relevant for legal purposes. It is not clear how aware doctors are of the importance of timely, thorough and accurate completion of the J88 form.

Final-year medical students at Stellenbosch University, Western Cape, South Africa, spend their Community Health and Family Medicine rotation in groups at primary care facilities. At a community health centre (CHC) in Cape Town, the medical manager brought a perceived problem concerning the management of assault victims who wish to make a case to the attention of the students allocated there. The problem was related to the protocol for obtaining, completion and further management of the $\mathrm{J} 88$ form. There seems to be a lack of clarity concerning each party's role in the process, which sometimes results in the victim terminating the case before completion. The student group decided to tackle this issue by performing a quality improvement cycle at the $\mathrm{CHC}$ and developing a standard operating procedure for the doctors working in casualty to follow.

Not only are the logistics of the J88 form important; how the doctor fills in the form is also critical. The content of the form should be of the highest quality in terms of accuracy and thoroughness. A training session on how to fill in each section correctly was hosted for the doctors at the CHC. (The need for training of locum doctors still exists, as the after-hours trauma services are covered by locum staff for a third of the time.) The Forensic Medicine Department at Stellenbosch University has also agreed to include training on the J88 during the 'wound description' lectures in the undergraduate forensics course. Current and future doctors need to be equipped with the knowledge and skills necessary to fill in the J88 form. Most importantly, doctors need to adopt a willing attitude and make themselves available to help.

This small project revealed an important gap in medical education and practice. Research is needed to delineate the problem and determine its extent. Barriers to the completion of J88 forms should be identified and actively addressed. It is essential that all the parties involved are aware of their specific roles in the process, and policy should be formulated to clarify these roles. As doctors, we need to remember that we play a vital part in ensuring that justice is served in cases of assault and rape, and we should take our role in the process seriously. Healthcare practitioners have an obligation not only to individual patients, but also to society as a whole. ${ }^{[4]}$ Let us join the fight against violence and work for 'justice through the J88'.

\section{Kirsten Rowe}

Hendrik Botha

Student interns, Faculty of Medicine and Health Sciences, Stellenbosch University, Cape Town, South Africa

kirstenrowe@gmail.com

\section{Hassan Mahomed}

Division of Community Health, Department of Interdisciplinary Sciences, Faculty of Medicine and Health Sciences, Stellenbosch University, Cape Town, South Africa

\section{Arina Schlemmer}

Division of Family Medicine and Primary Care, Department of Interdisciplinary Sciences, Faculty of Medicine and Health Sciences, Stellenbosch University, Cape Town, South Africa

\footnotetext{
South African Police Service. Crime Statistics Overview RSA 2011/2012. 2012. http://www.saps.gov.za/ statistics/reports/crimestats/2012/downloads/crime_statistics_presentation.pdf (accessed 19 March 2013). statistics/reports/crimestats/2012/downloads/crime_statistics_presentation.pdf (accessed 19 March 2013).
2. Mogale RS, Burns KK, Richter S. Violence against women in South Africa: policy position and recommendations. 2012. http://vaw.sagepub.com/content/18/5/580 (accessed 22 April 2013).

3. Müller K, Saayman G. Clinical forensic medicine: Completing the Form J88 - what to do and what not to do. South African Family Practice 2003;45(8):39-43. http://www.safpj.co.za/index.php/safpj/article/ view/1886/2407 (accessed 19 March 2013).

4. Health Professions Council of South Africa. General Ethical Guidelines for the Health Care Professions. 2008. http://www.hpcsa.co.za/downloads/conduct_ethics/rules/generic_ethical_rules/
} booklet_1_guidelines_good_prac.pdf (accessed 19 March 2013). 